OPINIÃO

OPINION

\title{
PROCOT - Desenvolvimento, resultados e avanços do Programa de Cooperação Técnica do Ministério da Saúde para informações técnico-econômicas em dispositivos médicos
}

\author{
PROCOT - Development, results and advances of the \\ Technical Cooperation Program of the Ministry of Health for \\ technical and economic information on medical devices
}

Murilo Contó

DOI: 10.21115/JBES.v9.suppl1.96-102

Palavras-chave:

dispositivos médicos, equipamentos, tecnologia biomédica, economia da saúde

\section{RESUMO}

Objetivo: Devido à escassez de evidências e de informações técnico-econômicas disponíveis, avaliar dispositivos médicos representa um trabalho desafiador que deve abordar características particulares que afetam diretamente a relação custo-efetividade. Informações econômicas são necessárias e objeto de análise durante várias etapas até o acesso ao paciente. Para aprimorar a captação de informações técnico-econômicas utilizadas pelo Ministério da Saúde, foi criado o Programa de Cooperação Técnica PROCOT. Métodos: Relato da experiência do autor enquanto idealizador e desenvolvedor do programa e buscas às páginas eletrônicas do Ministério da Saúde e Agência Nacional de Vigilância Sanitária (ANVISA). Resultados: O Ministério da Saúde repassa recursos financeiros para o financiamento de equipamentos que ocorre a partir de solicitações apresentadas em forma de propostas de instituições vinculadas ao SUS. A avaliação técnica-econômica busca a compatibilidade entre a densidade tecnológica do dispositivo e o valor requerido, sendo fundamental a utilização de informações oriundas do mercado para subsidiar a aprovação das propostas. O PROCOT visa à captação antecipada dessas informações do mercado nacional, organizando um banco de dados de fácil acesso. As empresas colaboradoras são divulgadas na internet e realizam palestras como um programa de atualização em tecnologias em saúde aos analistas do Ministério. A velocidade e a qualidade dos pareceres técnico-econômicos foram aprimoradas, repercutindo na aprovação mais célere dos repasses para a oferta de tecnologias ao Sistema Único de Saúde (SUS). Conclusão: O PROCOT é uma experiência exitosa que pode expandir seu escopo de tecnologias e usuários, servindo como modelo para a criação de uma rede internacional para intercâmbio de informações e avaliações técnico-econômicas em dispositivos médicos.

\section{Keywords:}

medical devices, equipment, biomedical technology, health economics

\begin{abstract}
Objective: Due to the scarcity of available evidence and technical-economic information, evaluating medical devices represents challenging work that must address particular characteristics that directly affect the cost-effectiveness ratio. Economic information is required and subject to analysis during several steps until access to the patient. To improve the collection of technical-economic information used by the Ministry of Health, the Technical Cooperation Program PROCOT was created. Methods: Reporting the author's experience as the creator and developer of the program and search the electronic pages of the Ministry of Health and National Health Surveillance Agency (ANVISA). Results: The Ministry of Health transfers financial resources for financing equipment that occurs from requests submitted in the form of proposals from institutions linked to Unified Health System (SUS). The technical-economic evaluation seeks to ensure compatibility between the technological

Recebido em: 05/08/2017. Aprovado para publicação em: 29/08/2017

1. Consultor Nacional em Gestão e Avaliação de Tecnologias em Saúde da Unidade Técnica de Medicamentos e Tecnologias em Saúde. Organização Pan-Americana de Saúde/Organização Mundial de Saúde, Brasília, DF, Brasil.

Financiamento: Não foram recebidos quaisquer auxílios sob a forma de financiamento para a realização deste trabalho.

As opiniões expressas representam unicamente a opinião do autor sobre os temas abordados, não se estendendo às instituições para as quais ele atua como colaborador.

Conflito de interesse: $\mathrm{O}$ autor declara não existir qualquer conflitos de interesse.

Autor correspondente: Murilo Contó. Q301, Conjunto 14, Lote 2-4, Ap. 702. Brasília, DF, Brasil. CEP: 71901-270. Telefone: +55 (61) 99974-2184. E-mail: muriloconto@terra.com.br
\end{abstract}


density of the device and the required value, and it is essential to use information from the market to subsidize the approval of proposals. PROCOT aims to capture this information early from the national market, organizing a data base easily accessible. Collaborating companies are publicized on the Internet and hold presentations as a program to upgrade on health technologies to Ministry analysts. The speed and quality of technical-economic advices were improved, impacting on the faster authorization of the transferences for the supply of technologies to SUS. Conclusion: PROCOT is a successful experiment that can expand its scope of technologies and users, serving as a model for the creation of an international network for the exchange of technical-economic information and evaluations of medical devices.

\section{Introdução}

A obtenção de informações claras e precisas sobre aspectos técnicos e econômicos relacionados a dispositivos médicos para subsidiar processos de Avaliação de Tecnologias em Saúde (ATS) tem sido um grande desafio para especialistas da área e também para gestores de serviços de saúde em geral. A assimetria de informações envolvendo dispositivos médicos contribui para que muitas distorções ocorram, dentre elas a elevada variação de preços praticados num mesmo mercado, que pode resultar em diferenças de até $1000 \%$ para um mesmo produto (Brasil, 2015).

A escassez de evidências científicas disponíveis na literatura quando comparada a outras tecnologias é outro fato que torna a avaliação de dispositivos médicos um trabalho desafiador. Além disso, há de se observar características particulares aos dispositivos, tais como curva de aprendizagem, tecnologia embarcada e infraestrutura necessária, que possuem uma influência mais significativa nos custos e na efetividade esperada do que a influência observada em medicamentos (Brasil, 2013).

Em razão dessas dificuldades, muitas avaliações sobre procedimentos terapêuticos e diagnósticos que requerem obrigatoriamente a utilização de algum equipamento não possuem uma abordagem com amplitude suficiente para dar cobertura a essas questões. Estudos de custo-efetividade e de impacto orçamentário numa ATS que envolva dispositivos médicos devem contemplar todo o cenário envolvido na sua utilização, caso contrário, uma lacuna perigosa poderá ser deixada, aumentando o nível de incertezas na tomada de decisão. Equipamentos como o tomógrafo computadorizado, o acelerador linear para radioterapia e a ressonância magnética podem ter significativos valores associados à utilização que, se não forem adequadamente estimados, podem representar desvios consideráveis na qualidade da recomendação. A estimativa desses custos associados ao ciclo de vida do equipamento é denominada Custo Total de Propriedade (ou Total Cost of Ownership), muito utilizada na gestão de equipamentos e que pode ser traduzida como a somatória de toda a cadeia de custos associada ao equipamento, desde a aquisição até o descarte, incluindo todas as despesas decorrentes da instalação, operação e manutenção (Brasil, 2013). Esse tipo de abordagem em relação aos custos de utilização de um equipamento tem impacto direto na sustentabilidade do serviço e na manutenção adequada dos parâmetros que asseguram a efetividade desejada durante toda a sua vida útil.

Informações técnicas e econômicas são tão relevantes na tomada de decisão quanto as evidências científicas são para demonstrar a eficácia e a segurança da tecnologia. São subsídios importantes para proporcionar escolhas mais acertadas tanto em nível macro como, por exemplo, numa incorporação para todo o Sistema Único de Saúde (SUS), como também em nível intermediário e micro, quando o gestor de determinado serviço de saúde ou mesmo o profissional de saúde deve decidir qual tecnologia será adquirida e aplicada aos seus pacientes.

De modo geral, as informações técnicas e econômicas relacionadas a dispositivos médicos são mandatórias em todos os processos de incorporação tecnológica em saúde no país e objeto de análises durante as diversas etapas em que a tecnologia é submetida até que esteja efetivamente disponível para o paciente (Figura 1). Inicia pelo registro na Agência Nacional de Vigilância Sanitária (ANVISA), onde, conforme a RDC no 185 de 2006, a empresa solicitante deve informar a intenção de preço a ser praticado no mercado. Na Agência Nacional de Saúde Suplementar (ANS) é solicitada a incorporação no rol dos planos de saúde para reembolso aos prestadores de serviços das operadoras privadas. Também passa pelo processo de incorporação no SUS, sendo submetida via demanda externa (empresas, associações, hospitais etc.) ou interna (instituições públicas ligadas ao SUS) ao Departamento de Gestão e Incorporação de Tecnologias em Saúde (DGITS) do Ministério da Saúde para deliberações e consultas públicas da Comissão Nacional de Incorporação de Tecnologias no SUS (CONITEC). Quando a recomendação final da CONITEC é favorável pela incorporação, passa para a disponibilização no SUS, tendo seus valores de reembolso ou de financiamento informados no SIGTAP - Sistema de Gerenciamento da Tabela de Procedimentos, Órteses, Próteses e Materiais Especiais, que gerencia informações sobre dispositivos de uso individual, ou no SIGEM - Sistema de Gerenciamento de Equipamentos e Materiais permanentes financiáveis para o SUS, que gerencia informações sobre equipamentos destinados ao uso coletivo.

No caso dos equipamentos financiáveis pelo Ministério da Saúde para estabelecimentos assistenciais vinculados ao SUS, a necessidade de se manter informações técnico-econômicas sempre atualizadas é imprescindível para garantir que 


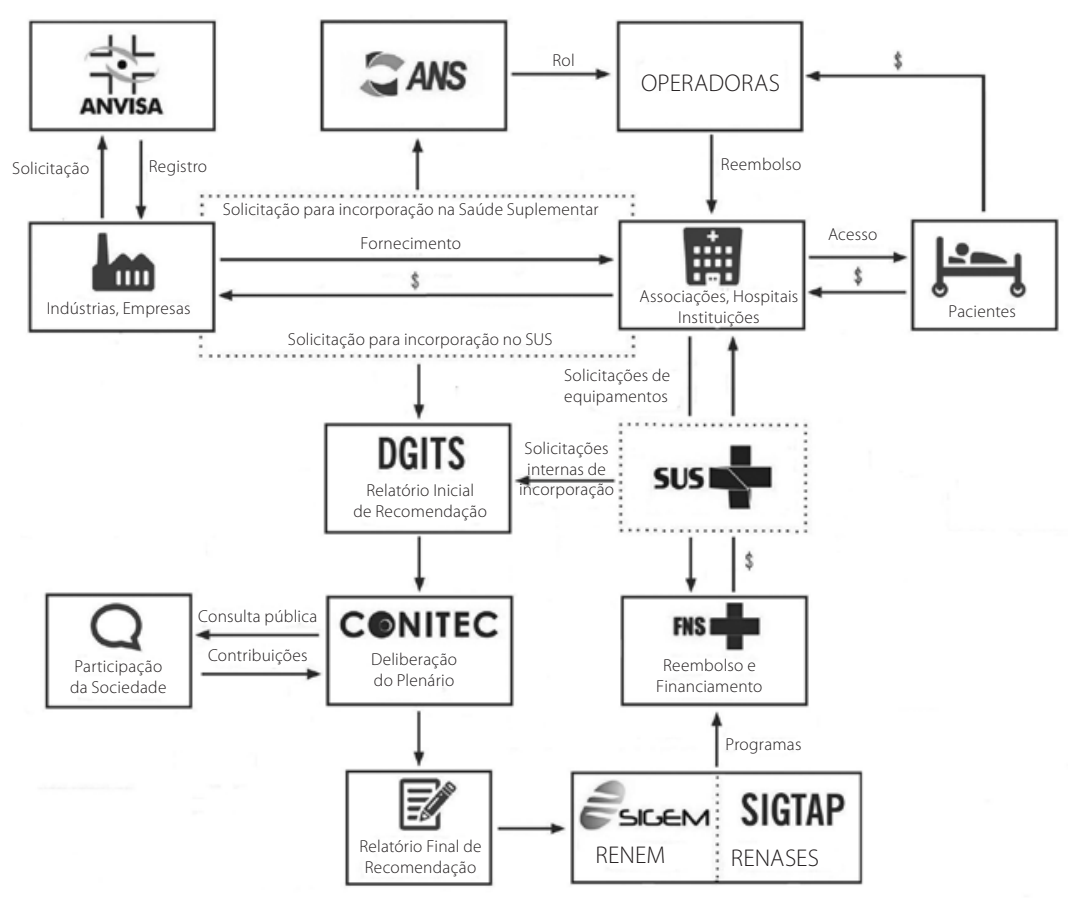

Figura 1. Fluxos de incorporação e acesso envolvendo dispositivos médicos no Brasil (elaboração própria).

as avaliações realizadas pelo Fundo Nacional de Saúde (FNS), relativas a solicitações de financiamentos, sejam realizadas sempre em observância às diferentes realidades mercadológicas e em conformidade com a complexidade tecnológica de cada equipamento.

Para promover tais avaliações com celeridade e pautadas por informações obtidas de forma sistemática e criteriosa, o Ministério da Saúde instituiu o PROCOT - Programa de Cooperação Técnica - para a captação de informações técnicas e econômicas de equipamentos por meio da participação voluntária de empresas fabricantes e de distribuidoras instaladas no território nacional (Brasil, 2017a).

Este artigo tem por objetivo descrever o processo de desenvolvimento e implementação do PROCOT, apontando os principais resultados obtidos em quase dez anos de funcionamento do programa e possibilidades de avanços, tanto no que tange às tecnologias contempladas, quanto em relação a novos potenciais usuários, como pesquisadores, gestores e especialistas de diversas regiões.

\section{Métodos}

A elaboração foi pautada por normas e políticas disponibilizadas nas páginas eletrônicas do Ministério da Saúde, da ANVISA e do Ministério do Planejamento, Orçamento e Gestão, associadas ao uso de informações técnico-econômicas para investimentos e a promoção do acesso às tecnologias em saúde caracterizadas como dispositivos médicos no SUS. Também foram obtidos dados sobre o programa PROCOT por meio de documentos disponibilizados na página do FNS na internet. As assertivas sem referências bibliográficas vinculadas consistem no relato da experiência do autor que, entre os anos de 2008 e 2015, idealizou e atuou nos processos de implantação e gestão do PROCOT junto à equipe da Divisão de Equipamentos da extinta Coordenação-Geral de Investimentos em Saúde (atual Coordenação de Análise de Investimentos e Infraestrutura) do Ministério da Saúde.

A construção do PROCOT ocorreu a partir de 2008, tendo como ações estruturantes três iniciativas fundamentais:

1. A adoção de uma base de nomenclaturas padronizada em função do porte e complexidade dos equipamentos incorporados e necessários para a execução dos procedimentos previstos na RENASES - Relação Nacional de Ações e Serviços em Saúde no SUS;

2. Criação de um repositório eletrônico centralizado para arquivamento e busca de informações técnicas e econômicas relacionadas aos equipamentos; e

3. Elaboração de estratégias para divulgação do programa, visando uma ampla e contínua participação de empresas fabricantes e distribuidoras de equipamentos médicos.

\section{Discussão}

Todos os anos, o Ministério da Saúde destina significativos valores exclusivamente à aquisição de equipamentos e materiais permanentes por instituições públicas e privadas (sem 
fins lucrativos), de forma descentralizada, com o intuito de melhorar e ampliar a oferta tecnológica do SUS. No ano de 2016 cerca de R\$ 3 bilhões foram destinados exclusivamente para tais aquisições (Brasil, 2017b). Trata-se de um valor bastante representativo principalmente quando comparado a outros programas estratégicos do Ministério da Saúde e de forte apelo popular, tal como o Programa Mais Médicos, cuja destinação orçamentária prevista para sua implementação em 2016 foi de R\$ 2,9 bilhões, e o Programa Farmácia Popular, com orçamento previsto de R\$2,7 bilhões para o seu funcionamento, conforme a Lei de Diretrizes Orçamentárias de 2016 (Brasil, 2016).

Diante desses números, é evidente que a gestão e alocação desses recursos possui grande impacto, podendo representar grandes avanços na infraestrutura tecnológica do SUS.

Essa expressiva soma financeira é repassada por meio da formalização de projetos de investimentos apresentados em forma de propostas ao Ministério da Saúde por instituições vinculadas ao SUS. Para que o repasse aconteça, é necessária a aprovação da proposta, tanto sob o ponto de vista do mérito como também sob o ponto de vista técnico-econômico. As avaliações de mérito e técnico-econômica têm um papel fundamental na otimização desses recursos por estabelecer uma coerência e compatibilidade entre o perfil do estabelecimento assistencial, as especificações técnicas apresentadas (que determinam o porte e a complexidade tecnológica dos equipamentos), e os valores de financiamento solicitados (Brasil, 2017c).

Antes da criação do PROCOT em 2008, a prática rotineira das avaliações técnico-econômicas era baseada em buscas pontuais na internet por referências de preços ou no contato direto com distribuidores e fabricantes para o recebimento de cotações que servissem como subsídio para a emissão dos pareceres. Essa busca por informações só ocorria após o recebimento da proposta para análise, quando então o analista tomava conhecimento da relação de equipamentos pleiteados pela instituição. Essa ação puramente reativa gerava um entrave importante, pois, enquanto as informações econômicas não eram obtidas, as propostas permaneciam sem o devido parecer técnico-econômico, impedindo o repasse dos recursos e o consequente acesso da população à tecnologia, quando esta apresentava características favoráveis à incorporação junto aos serviços de saúde.

Um outro agravante era a forma de arquivamento dessas informações econômicas que, após serem obtidas, não eram alocadas em uma base de dados apropriada, permanecendo muitas vezes restritas apenas a um usuário: o próprio analista que realizou a busca. Essa ausência de um banco de dados compartilhado, associada à falta de um embasamento técnico mais harmonizado principalmente quanto às tecnologias mais complexas, acarretava na elevação da subjetividade das análises, pois não havia uma parametrização comum de re- ferências, tanto técnicas como econômicas, a toda equipe de analistas. Essa subjetividade significava que um equipamento de mesma especificação poderia ser aprovado em propostas distintas com valores bastante díspares em função da assimetria de informações oriundas das diferentes fontes de informação utilizadas.

Dada à diversidade de tecnologias disponíveis, ao ritmo acelerado de inovações e à grande variedade de especificações técnicas e configurações possíveis nos equipamentos, os analistas também se deparavam muitas vezes com dificuldades para identificar quais características realmente possuíam alguma relevância em relação aos desfechos esperados e quais delas representavam maior impacto no preço estimado.

Diante desse cenário, a implantação do PROCOT se iniciou com a realização de algumas ações estruturantes para a promoção de maior precisão e eficiência nos processos de avaliação de investimentos em equipamentos do Ministério da Saúde. Essas ações tinham por objetivo criar mecanismos de captação, arquivamento histórico e promoção do acesso rápido às informações técnicas e econômicas, fundamentais para a realização de avaliações tempestivas e adequadas à alocação e liberação de recursos de forma mais eficiente.

A primeira dessas ações foi um trabalho de padronização de nomenclaturas dos equipamentos. No início de 2008, a lista de equipamentos financiáveis pelo Ministério da Saúde para o SUS possuía cerca de 3.000 termos, muitos deles redundantes, representando o mesmo equipamento, mas com pequenas variações no nome, como, por exemplo, "bomba infusora" e "bomba de infusão". Após o trabalho de padronização, a relação de equipamentos financiáveis foi reduzida para aproximadamente 900 itens, assegurando que cada tipo de equipamento possuía apenas um termo válido para sua identificação. Critérios técnicos foram estabelecidos para a inclusão de novos termos, no intuito de evitar inserções desnecessárias à lista. Este trabalho resultou posteriormente na criação da RENEM - Relação Nacional de Equipamentos e Materiais permanentes financiáveis para o SUS (Contó \& Petramale, 2015).

O passo seguinte foi a criação de repositórios eletrônicos específicos para o arquivamento das informações obtidas, onde cada termo da relação de equipamentos passou a ter uma pasta eletrônica correspondente. $\mathrm{O}$ acesso às pastas passou a ser promovido por links vinculados a cada nomenclatura e os repositórios foram alocados no servidor central do departamento, permitindo que todos os analistas tivessem acesso a uma única base de dados tanto para consulta como para inserção de suas contribuições.

Por fim, foram delineadas estratégias e regras do programa quanto à participação das empresas e as contrapartidas necessárias para promover a maior adesão possível. A estratégia de divulgação ocorreu por meio de entidades ligadas à 
indústria de equipamentos médicos no país, a participação em feiras e congressos da área, assim como o contato direto com fornecedores. Como atrativos à adesão, foi definida uma agenda para a realização de palestras técnicas a serem proferidas pelas empresas participantes sobre suas linhas de produtos, desde que suas informações técnicas e econômicas tivessem sido enviadas e validadas. As palestras técnicas proferidas aos analistas do Ministério da Saúde e de outros órgãos federais, como a ANVISA e a Empresa Brasileira de Serviços Hospitalares (EBSERH), abriram um importante espaço de diálogo entre técnicos do governo e técnicos da indústria para eliminar quaisquer dúvidas técnicas e econômicas associadas aos equipamentos. Outro atrativo importante para as empresas se tornarem colaboradoras do PROCOT foi a vinculação de seus nomes e dados de contato aos equipamentos financiáveis divulgados pelo sistema SIGEM na internet. Essa vinculação estabeleceu um tipo de catálogo eletrônico, totalmente aberto ao acesso público, para consulta dos equipamentos e suas opções no mercado. As informações divulgadas ao público em geral são genéricas, resultado da compilação das informações específicas de cada produto enviadas pelas empresas que são de uso interno e exclusivo do Ministério da Saúde.

A divulgação do programa passou a ocorrer sistematicamente a cada início de ano, quando o banco de dados é renovado e há a necessidade de alimentá-lo com informações atualizadas antes do período de apresentação de novas propostas de investimentos ao Ministério da Saúde.

Buscando dar ampla publicidade e transparência ao programa, todas as regras do PROCOT foram inseridas em um manual permanentemente atualizado e disponibilizado na página do FNS na internet (http://portalfns.saude.gov.br/procot).

\section{Resultados}

A implantação do PROCOT trouxe avanços importantes ao processo de avaliações técnico-econômicas e consequentemente à liberação de investimentos para a aquisição de equipamentos para o SUS.

A criação de uma base de dados de preços, especificações técnicas e de outras informações técnico-científicas relacionadas aos equipamentos promoveu aos analistas de projetos uma ferramenta segura e facilitadora para acessar com rapidez todas as informações disponíveis e necessárias para as avaliações.

O reflexo mais imediato foi a velocidade com que as análises de investimentos passaram a ser realizadas, pois a presença de informações técnicas e econômicas, já disponíveis para consulta imediata antes da submissão das propostas, eliminou em grande parte a necessidade de buscas empíricas a cada proposta recebida, aumentando o tempo de dedicação dos analistas à efetiva avaliação dos projetos.
A segurança das avaliações e a redução da subjetividade também foram avanços alcançados com a centralização das informações e com as palestras técnicas. A centralização e unificação da base de dados possibilitou o acesso de todos os analistas a mesma fonte de consulta e a um número maior de informações, disponível graças à cooperação mútua de todos os membros da equipe, responsáveis por alimentar os repositórios. As palestras técnicas tiveram um importante papel complementar às informações disponibilizadas, pois, quanto mais complexo o equipamento, maiores são as especificidades em características técnicas e tecnologias embarcadas, exigindo mais conhecimento especializado por parte dos analistas. Assim, o programa de palestras técnicas do PROCOT funcionou como um programa de capacitação e atualização contínua em tecnologias em saúde aos analistas técnicos do Ministério da Saúde e de outros órgãos do governo, nos quais os conhecimentos adquiridos se refletiram também na maior rapidez e qualidade das análises, proporcionando respostas mais céleres para a aquisição de equipamentos à saúde pública de todo o país.

A divulgação das empresas colaboradoras no sistema SIGEM com links de acesso às suas respectivas páginas eletrônicas disponibilizou ao público em geral informações sobre os equipamentos financiáveis para o SUS e suas opções no mercado, auxiliando técnicos e gestores de todo o país na elaboração de projetos de investimentos em infraestrutura tecnológica para a saúde. Segundo dados disponibilizados pelo FNS, o PROCOT contava até julho de 2017 com 195 empresas ativas como colaboradoras (Brasil, 2017d). Isso significa que todas essas empresas vêm contribuindo com o programa, fornecendo antecipadamente informações técnicas e econômicas sobre sua linha de produtos.

Outros processos de trabalho referentes aos projetos de investimentos também foram fortalecidos, como as análises de reformulações e remanejamentos de recursos de projetos em execução, análises de prestação de contas e respostas às demandas de órgãos de controle como o Tribunal de Contas da União (TCU) e a Controladoria Geral da União (CGU), que, em determinadas situações, solicitam pronunciamentos quanto aos preços praticados por instituições que receberam recursos públicos. Ao estabelecer um banco de dados com o histórico de informações técnicas e econômicas armazenadas e separadas por ano e por tipo de tecnologia, foi possível realizar pesquisas retrospectivas de preços de referência, fundamentando de forma mais conclusiva vários processos de prestação de contas e demandas destes e de outros órgãos públicos.

\section{Conclusão}

Pode-se afirmar que, após quase dez anos de funcionamento, o PROCOT foi e continua sendo uma iniciativa exitosa que 
trouxe um ganho significativo aos processos de investimentos em equipamentos do Ministério da Saúde, colaborando ainda com outras áreas afins que também necessitavam de uma melhor sistemática de captação e acesso às informações técnico-econômicas para apoiar suas atividades. Sua importância e relevância foram reconhecidas quando, por meio da Portaria GM/MS no 3.134 de 17 de dezembro de 2013, o PROCOT foi instituído como um programa oficial do Ministério da Saúde.

Esse êxito, porém, não significa que o programa esteja perfeito, pois existem muitas oportunidades de melhorias e avanços que devem ser consideradas. Uma dessas oportunidades é a possibilidade de se promover uma participação mais representativa de empresas colaboradoras por todo o território nacional. A elevada concentração de empresas participantes nas regiões sul e sudeste (Figura 2) evidentemente reflete o maior desenvolvimento industrial e mercadológico dessas regiões, mas pode gerar uma parametrização de valores imprecisa ou inexequível para outras regiões do país, como, por exemplo, a região norte, onde reconhecidamente as questões logísticas são mais complexas e onde, até julho de 2017, não havia nenhuma empresa participante. Além da questão logística, aspectos tributários que variam de Estado para Estado também podem interferir na parametrização de preços, reforçando a necessidade de uma participação mais representativa em nível nacional, que poderia mitigar a influência causada por essa elevada concentração regional de empresas participantes.

Outra possibilidade de melhoria refere-se ao escopo do programa que, atualmente, contempla apenas equipamentos e materiais permanentes. Como já mencionado, a assimetria de informações em dispositivos médicos gera distorções bastante onerosas, fazendo com que os preços de um mesmo dispositivo possam ter variações extremas. Essa assimetria de informações se espalha por toda cadeia de acesso à tecnologia, tendo muitas vezes significativas repercussões orçamentárias, tanto em processos regulares de dispensação, quanto em processos de judicialização. Assim, uma possível expansão do escopo das tecnologias, contemplando dispositivos médicos que possuem maior valor agregado e maior impacto orçamentário para os sistemas de saúde, como ocorre com os dispositivos implantáveis, seria bastante interessante para os órgãos responsáveis pelos processos de regulação, incorporação e reembolso.

Por fim, o PROCOT poderia servir como modelo para um programa similar ampliado para a participação de outros colaboradores e instituições de outros países, estabelecendo um sistema de intercâmbio de preços e avaliações internacionais de dispositivos médicos. Em algumas redes de trabalho internacionais, como a Rede de ATS das Américas (RedETSA) e o Grupo Regional de Agências Reguladoras de Dispositivos Médicos, às quais a OPAS - Organização

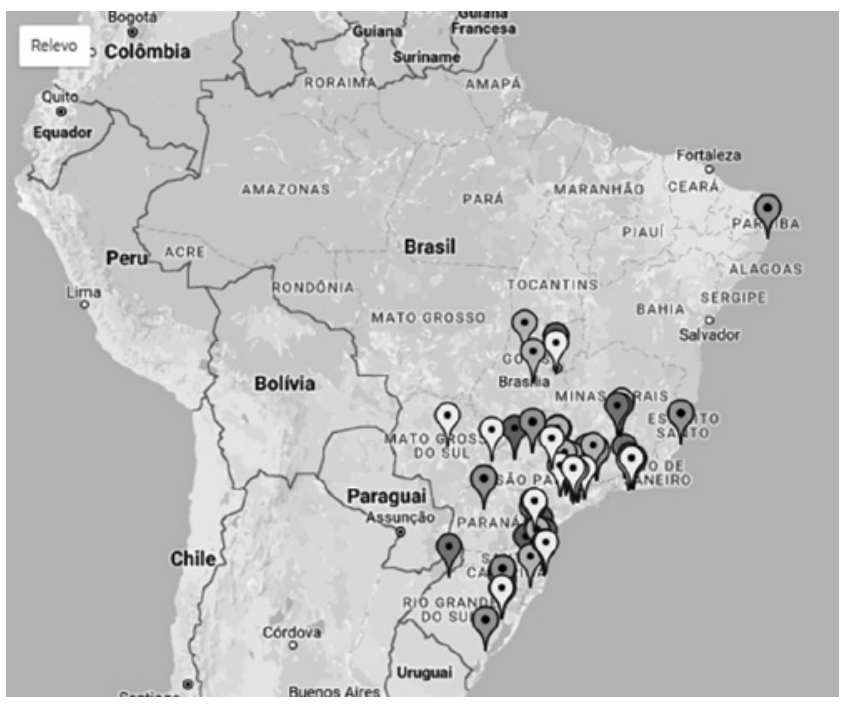

Figura 2. Distribuição territorial de empresas participantes do PROCOT (Brasil, 2017e).

Pan-Americana de Saúde - exerce um papel de secretariado-executivo, o intercâmbio de informações e execução de projetos em cooperação já ocorrem com boa sinergia de esforços. A criação de um programa internacional de cooperação para captação e compartilhamento de informações técnicas e econômicas nos moldes do PROCOT e das redes de trabalho já existentes poderia ser também implementado em âmbito continental, utilizando plataformas já disponíveis como o Cisco WebExं, utilizado para os webinars da RedETSA, e a PRAIS - Plataforma Regional sobre Acesso e Inovação para Tecnologias em Saúde, mantida pela OPAS para compartilhamento de informações.

Essa iniciativa representaria mais um grande avanço para todos os países da região, fortalecendo suas capacidades em estabelecer políticas voltadas a inovação, regulação, incorporação e acesso às tecnologias em saúde para suas populações, colaborando ainda para uma maior eficiência e sustentabilidade de seus sistemas.

\section{Agradecimentos}

Ao Eng. Marcos Lucatelli, pelo apoio à implantação do programa quando ainda era apenas uma ideia; a todos os colegas do Ministério da Saúde que participaram da construção, aprimoramento e gestão do PROCOT; e à colega Érika Pacheco, pela arte aplicada na ilustração deste artigo.

\section{Referências bibliográficas}

Brasil. Ministério da Saúde. Relatório Final do Grupo de Trabalho Interinstitucional sobre órteses, próteses e materiais especiais (GTIOPME), instituído pela Portaria Interministerial n 38 , de 8 de janeiro de 2015; 2015. p.78. 
Brasil. Ministério da Saúde. Secretaria de Ciência, Tecnologia e Insumos Estratégicos. Departamento de Ciência e Tecnologia. Diretrizes metodológicas: elaboração de estudos para avaliação de equipamentos médicos assistenciais/Ministério da Saúde, Secretaria de Ciência, Tecnologia e Insumos Estratégicos, Departamento de Ciência e Tecnologia. Brasília: Ministério da Saúde, 2013.96 p.

Brasil. Ministério da Saúde. Secretaria-Executiva. Fundo Nacional de Saúde. O PROCOT, 2017a. Disponível em: <http://portalfns.saude.gov.br/ apresentacao>. Acessado em: 18/07/2017.

Brasil. Ministério da Saúde. Secretaria-Executiva. Fundo Nacional de Saúde. Cenário das Atualizações do Programa de Cooperação Técnica: 2017, 2017b. Disponível em: <http://portalfns.saude.gov.br/in>. Acessado em: 18/07/2017.

Brasil. Ministério do Planejamento, Orçamento e Gestão. Secretaria de Orçamento Federal. Lei Orçamentária Anual, Volume II, 2016. Disponível em: <http://www.orcamentofederal.gov.br/clientes/portalsof/portalsof/ orcamentos-anuais/orcamento-2016/loa/loa-2016-volume-ii.pdf> Acessado em: 23/07/2017.
Brasil. Ministério da Saúde. Secretaria-Executiva. Cartilha de apresentação de propostas ao Ministério da Saúde: 2017 / Ministério da Saúde, SecretariaExecutiva. Brasília: Ministério da Saúde, 2017c. 202 p. ISBN 978-85-3342478-4. Disponível em: <http://www.fns2.saude.gov.br/documentos/ cartilha.pdf> Acessado em: 18/07/2017.

Contó M, Petramale CA. RENEM - Relação Nacional de Equipamentos e Materiais Permanentes Financiáveis para o SUS. Revista Eletrônica Gestão \& Saúde. 2015;6(Supl. 4):3213-24. Acessado em: 18/07/2017.

Brasil. Ministério da Saúde. Secretaria-Executiva. Fundo Nacional de Saúde. Empresas Colaboradoras do PROCOT: 2017, 2017d. Disponível em: <http://portalfns.saude.gov.br/images/banners/Procot/FORNECEDOR_ ITENS_JULHO_v_final.pdf. Acessado em: 06/08/2017.

Brasil. Ministério da Saúde. Secretaria-Executiva. Fundo Nacional de Saúde. Empresas Colaboradoras do PROCOT: 2017, 2017e. Disponível em: <http://portalfns.saude.gov.br/mapa>. Acessado em: 06/08/2017. 